\title{
Abnormal torsion and helical flow patterns of the neo-aorta in hypoplastic left heart syndrome assessed with 4D-flow MRI
}

\author{
Dominik Daniel Gabbert ${ }^{1}$, Patrick Trotz ${ }^{1}$, Arash Kheradvar $^{2} \wedge$, Michael Jerosch-Herold ${ }^{3} \wedge$, Jens Scheewe ${ }^{1,4}$, \\ Hans-Heiner Kramer ${ }^{1 \wedge}$, Inga Voges ${ }^{1 \wedge}$, Carsten Rickers ${ }^{1 \wedge}$ \\ ${ }^{1}$ Department of Congenital Heart Disease and Pediatric Cardiology, DZHK (German Center for Cardiovascular Research), partner site Hamburg/ \\ Kiel/Lübeck, University Hospital Schleswig-Holstein, Kiel, Germany; ${ }^{2}$ The Edwards Lifesciences Center for Advanced Cardiovascular Technology, \\ University of California, CA, Irvine, USA; ${ }^{3}$ Harvard Medical School, Brigham and Women's Hospital, Boston, MA, USA; ${ }^{4}$ Department of Cardiac \\ and Vascular Surgery, University Hospital Schleswig-Holstein, Kiel, Germany \\ Contributions: (I) Conception and design: DD Gabbert, A Kheradvar, M Jerosch-Herold, HH Kramer, C Rickers; (II) Administrative support: DD \\ Gabbert, C Rickers; (III) Provision of study materials or patients: C Rickers, HH Kramer; (IV) Collection and assembly of data: C Rickers, I Voges, \\ DD Gabbert; (V) Data analysis and interpretation: DD Gabbert, A Kheradvar, M Jerosch-Herold, C Rickers; (VI) Manuscript writing: All authors; \\ (VII) Final approval of manuscript: All authors. \\ Correspondence to: Prof. Dr. Carsten Rickers. Universitäres Herz- und Gefäßzentrum UKE Hamburg, Martinistraße 52, 20251 Hamburg, Germany. \\ Email: c.rickers@uke.de.
}

Background: The Norwood procedure is the first stage of correction for patients with hypoplastic left heart syndrome (HLHS) and may lead to an abnormal neoaortic anatomy. We prospectively studied the neoaorta's fluid dynamics and the abnormal twist of the neoaorta by MRI examinations of HLHS patients in Fontan circulation. This study for the first time investigates the hypothesis that the neoaorta twist is associated with increased helical flow patterns, which may lead to an increased workload for the systemic right ventricle (RV) and ultimately to RV hypertrophy.

Methods: A group of forty-two HLHS patients with a median age of 4.9 (2.9-17.0) years, at NYHA I was studied along with a control group of eleven subjects with healthy hearts and a median age of 12.1 (4.0-41.6). All subjects underwent MRI of the thoracic aorta including ECG-gated 2D balanced SSFP cine for an axial slice stack and 4D-flow MRI for a sagittal volume slab covering the thoracic aorta. The twist of the neoaortic arch was quantified by the effective geometric torsion, defined as the product of curvature and geometric torsion. Fluid dynamics and geometry in the neoaorta, including the flow helicity index, were evaluated using an in-house analysis software (MeVisLab-based). Myocardial mass of the systemic ventricle at end-diastole was estimated by planimetry of the short-axis stack.

Results: Compared to the control group, the neoaorta in the HLHS patients shows an increased twist $(\mathrm{P}=0.04)$ and higher peak helicity density $(\mathrm{P}=0.03)$. The maximum helicity density was correlated with maximum effective torsion of the ascending neoaorta $(\mathrm{P}<0.001)$. The degree of maximum twist correlated with the increase in RV myocardial mass $(\mathrm{P}<0.01)$.

Conclusions: This study shows that the abnormal twist of the neoaortic arch in HLHS patients is associated with abnormal helical flow patterns, which may contribute to increased RV afterload and may adversely affect the systemic RV by stimulation of myocardial hypertrophy. These findings suggest that further improvements of surgical aortic reconstruction, guided by insights from 4D-flow MRI, could lead to better neoaortic fluid dynamics in patients with HLHS.

Keywords: Hypoplastic left heart syndrome (HLHS); kinking; torsion; neoaorta; helicity; 4D flow MRI

\footnotetext{
^ ORCID: Dominik Daniel Gabbert, 0000-0001-7081-6345; Arash Kheradvar, 0000-0003-3864-1359; Michael Jerosch-Herold, 0000-00019018-6731; Hans-Heiner Kramer, 0000-0001-8275-0347; Inga Voges, 0000-0001-7406-8006; Carsten Rickers, 0000-0003-3734-3296.
} 
Submitted Sep 08, 2020. Accepted for publication Dec 08, 2020.

doi: $10.21037 / \mathrm{cdt}-20-770$

View this article at: http://dx.doi.org/10.21037/cdt-20-770

\section{Introduction}

In patients with hypoplastic left heart syndrome (HLHS), formation of a "neo-aorta" is an integral part of the Norwood procedure (Figure 1) that includes creation of an anastomosis of the native aorta to the former pulmonary trunk and reconstruction of the ascending aorta and transverse arch using a porcine pericardial graft $(1,2)$. This sophisticated aortic surgery leads to an abnormal neoaortic twist that potentially leads to abnormal fluid dynamics and increased ventricular afterload with subsequent myocardial hypertrophy and impaired ventricular function in the long run (3-5).

4D flow MRI has been shown to provide important information on fluid dynamics in the cardiovascular system (6). The agreement of 4D flow MRI with 2D phase contrast has been demonstrated previously (7). Abnormal fluid dynamics in the neoaorta of HLHS patients were quantified in a previous 4D-flow MRI study (8). Several other computational fluid dynamics (CFD) studies have also shown the impact of the aortic geometry on haemodynamics (9-11). A CFD study on aortic models with and without twist predicted a relationship between twist and helical flow patterns and motivated further studies on our patient cohort (10). No previous clinical MRI study quantified the relationship between aortic twist and helical flow patterns in HLHS patients' neoaorta.

The present study aims to investigate the neoaorta's abnormal twist and its consequences on fluid dynamics as well implications for the RV. We present the following article in accordance with the MDAR reporting checklist (available at http://dx.doi.org/10.21037/cdt-20-770).

\section{Methods}

\section{Patient data}

HLHS patients were enrolled for an MRI study after 3 stage palliation (12) at the Kinderherzzentrum Kiel, Germany. The three stages of surgical palliation involved: (I) modified Norwood procedure with a Blalock-Taussig (BT)-Shunt and an aortic arch reconstruction using a porcine pericardial patch augmentation (Figure 1), (II) superior cavo-atriopulmonary anastomosis "Hemi-Fontan" with enlargement of the left pulmonary artery (LPA), and stage (III) Total cavo- pulmonary anastomosis by creating an intraatrial fenestrated tunnel. The patient's ascent and parent's consent were obtained prior to any MRI study. Healthy controls were recruited from subjects ruled out for cardiovascular disease. The subjects' demographic characteristics are listed in Table 1 . The study was approved by the institutional ethics board of Kiel University's Faculty of Medicine (A168/07). The study was conducted in accordance with the Declaration of Helsinki (as revised in 2013).

\section{Data acquisition and reconstruction}

Cardiovascular MRI examinations were performed on a 3.0 Tesla MR system (Achieva 3.0T, TX-series, Philips Healthcare, Best, The Netherlands) with a 32-channel coil for cardiac imaging. Blood flow velocities were quantified with a time-resolved phase-contrast with three-dimensional anatomic coverage (4D-flow MRI) using retrospective ECG-gating and a temporal phase resolution of 30-40 ms. The velocity-encoding was set to values of $\mathrm{VENC}=150$ $200 \mathrm{~cm} / \mathrm{s}$ (corresponding to expected peak velocity) for velocity encoding without aliasing, which was $10 \%$ above the expected peak blood flow velocity.

Scanned data were acquired under free-breathing conditions using an MR navigator signal read-out for tracking of diaphragmatic motion with a respiratory drift correction of up to a maximum drift $2 \mathrm{~mm}$ per minute. The acceptance window of respiratory navigator of 4-8 $\mathrm{mm}$ relative to the end-expiratory position was applied to achieve a respiratory acceptance between $40 \%$ and $70 \%$ and a total acquisition time of 8-12 minutes. A $2.2 \mathrm{~mm}$ isovoxel resolution was used to resolve a stack volume with a $25 \mathrm{~cm} \times 25 \mathrm{~cm}$ field of view in sagittal orientation and a stack thickness of $6-9 \mathrm{~cm}$. The flip angle was $\alpha=10^{\circ}$, TR/ TE was $4.7-5.4 \mathrm{~ms} / 2.2-2.8 \mathrm{~ms}$ and sensitivity encoding (SENSE) factor for parallel imaging acceleration was 2. A non-symmetric 4-point phase contrast velocity encoding was used. A linear phase correction was applied to velocityencoded phase contrast data. The slab for 4D-flow MRI covered the aortic arch down to the diaphragm and the pulmonary arteries near the bifurcation (Figures 2,3).

ECG-gated measurement of the short axis stack using a $2 \mathrm{D}$ balanced SSFP cinesequence was used to quantify 

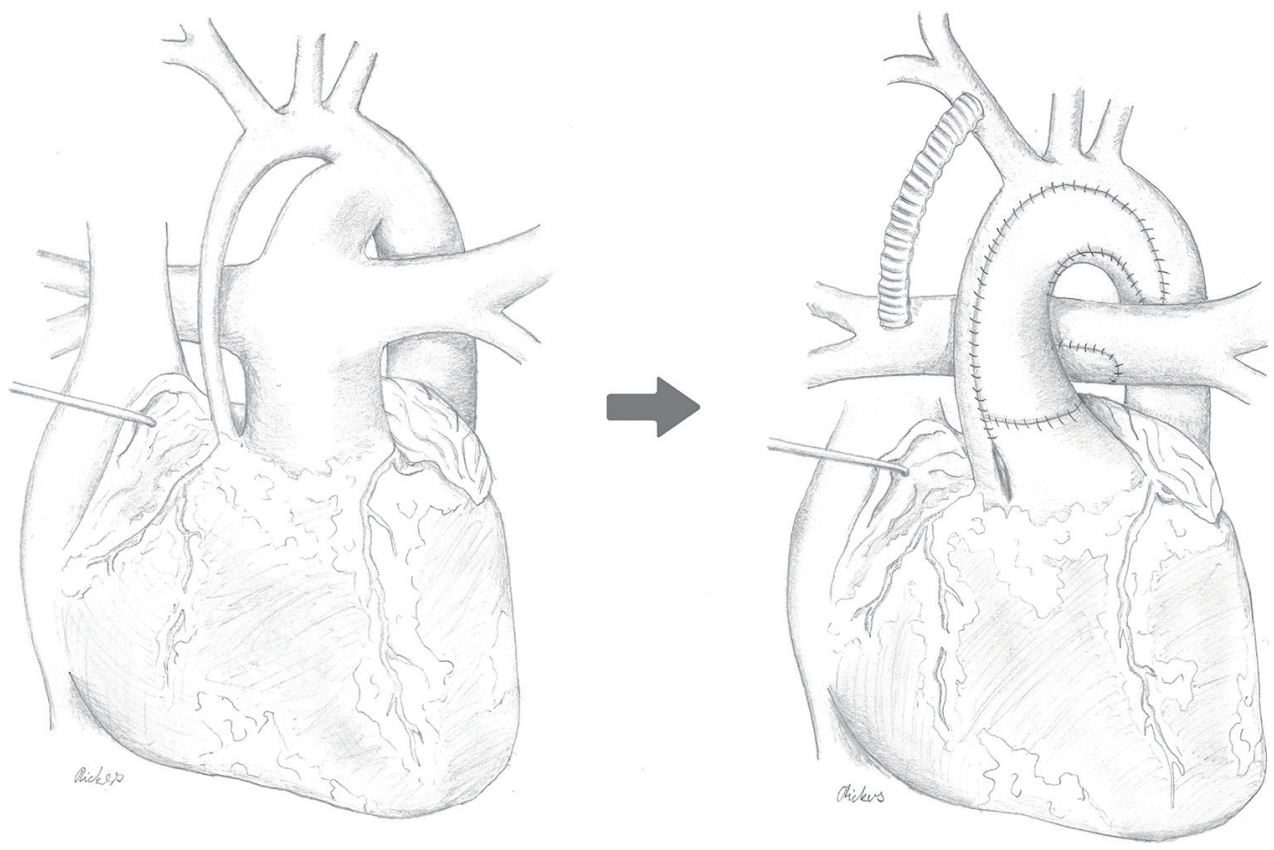

Figure 1 Schematic drawing of the Norwood procedure as the first step of palliating hypoplastic left heart syndrome (HLHS), which includes formation of a "neo-aorta" from the former pulmonary trunk and the native hypoplastic aorta. For reconstruction of the ascending aorta and transverse arch graft material is being used.

Table 1 Demographic characteristics of patients and healthy controls

\begin{tabular}{|c|c|c|c|}
\hline Parameter & HLHS subjects $(n=42)$ & Healthy controls $(n=11)$ & $P$ value \\
\hline \multicolumn{4}{|l|}{ Sex } \\
\hline Male & $39(69 \%)$ & $4(36.4 \%)$ & 0.09 \\
\hline Female & $13(31 \%)$ & $7(63.6 \%)$ & \\
\hline \multicolumn{4}{|l|}{ Age } \\
\hline At TCPC (years) & $2.55(1.7-5.1)$ & - & - \\
\hline At MRI (years) & $4.85(2.9-17)$ & $12.1(4-41.6)$ & 0.006 \\
\hline Weight (kg) & $23.84 \pm 12,93$ & $50.25 \pm 26.56$ & $<0.001$ \\
\hline Height (cm) & $116.65 \pm 22.65$ & $149.02 \pm 28.84$ & 0.002 \\
\hline \multicolumn{4}{|l|}{ HLHS subtype } \\
\hline MA/AA & $19(45 \%)$ & - & - \\
\hline MS/AA & $11(26 \%)$ & - & - \\
\hline MA/AS & $3(7 \%)$ & - & - \\
\hline MS/AS & $9(21)$ & - & - \\
\hline
\end{tabular}

HLHS, hypoplastic left heart syndrome. 

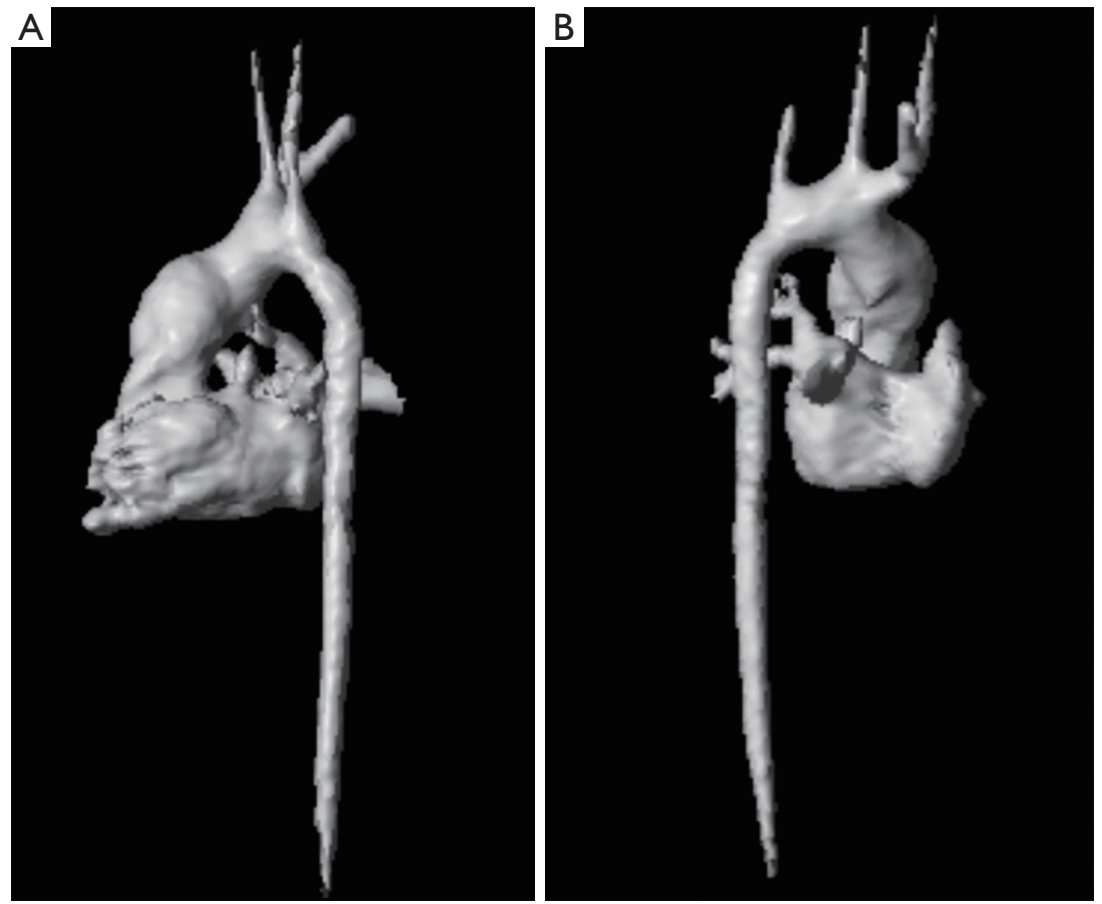

Figure 2 The complex aortic surgery for the Norwood procedure leads to an abnormal neoaortic twist as illustrated by this $3 \mathrm{D}$ reconstructing using 4D-flow MRI data.
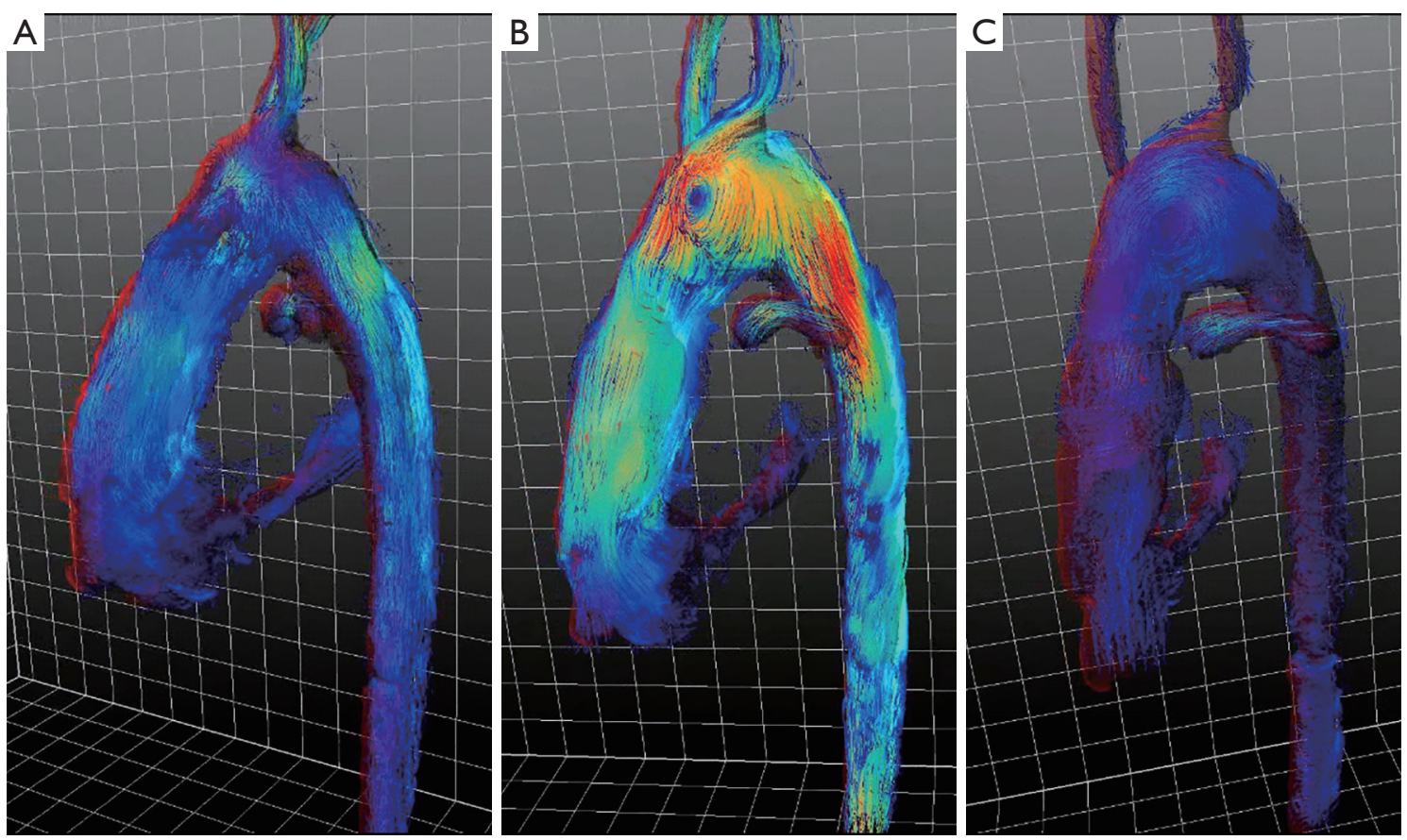

Figure 3 Visualization of blood flow by streamlines in the thoracic aorta of a HLHS patient with evident dilation of the aortic arch. (A) In early systole, the flow velocity is elevated near the aortic isthmus; (B) a pronounced helical flow pattern is visible in systole in the aortic arch, and in the descending aorta with a clockwise rotation, distinct from the normal flow pattern with counter-clockwise rotation in the descending aorta; (C) the helical flow pattern extends into diastole. HLHS, hypoplastic left heart syndrome. 


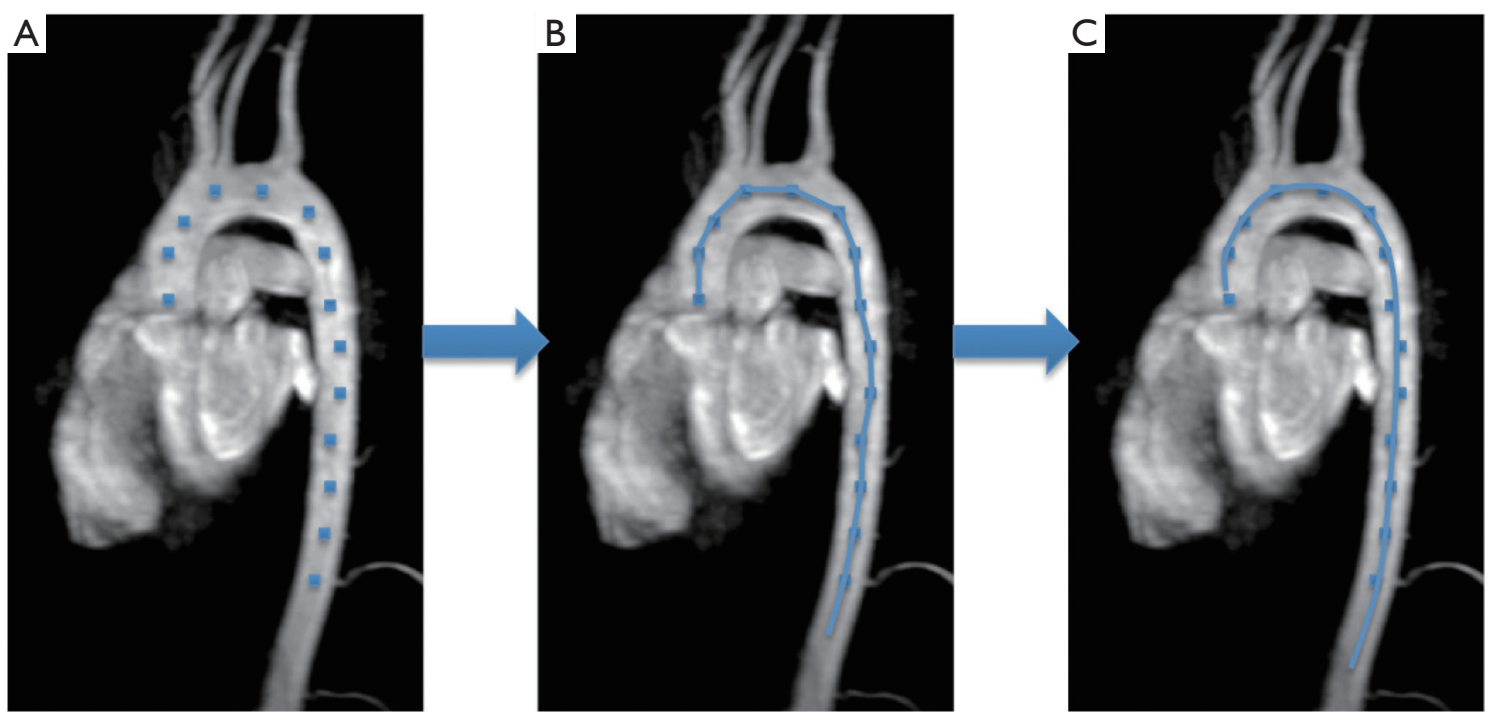

Figure 4 Schematic representation of the construction of the centerline. (A) Landmarks are set along the aorta between aortic valve and diaphragm, (B) which will be connected (C) as well as smoothed in the last step.

myocardial mass.

\section{Data analysis}

4D-flow MRI data of the HLHS patients and controls were acquired according to common guidelines (6) (Figure 3) and analyzed by an in-house analysis software (MeVisLabbased). Landmarks were set to define the centerline of the aorta between aortic valve and diaphragm (Figure 4A,4B). A curved vessel centerline was defined as the natural cubic spline through the landmarks (Figure $4 C$ ). The aorta's geometric characteristics including curvature and torsion were determined as a function of the position alongside the aortic centerline using the Frenet-Serret formulas. In this context, the term torsion refers to the geometric property of the centerline twist. An in-plane curvature has no such torsion, whereas a curvature which leaves a plane has torsion. The product of torsion and curvature, referred to as 'effective torsion', was computed. The aortic arch's position was determined by the most cephalic point on the centerline (closest to the patient's head). The sections proximal or distal to this reference point in the aorta are referred to as the ascending or descending neoaorta. The section of the aorta in between the aortic valve and the diaphragm is considered as thoracic aortic section.

A multi-planar reconstruction (MPR) with slice thickness of $2.2 \mathrm{~mm}$ provided blood flow data in planes, perpendicular to the aortic centerline (6). By integration over the MPR planes, helicity density was calculated as a function of the position along the aorta $r$ and time t:

$$
H_{d}(r, t)=\frac{\int u(x, t) \cdot \omega(x, t) d V_{r}}{V_{r}}
$$

where $V_{r}$ is the slice volume, $u$ is the velocity vector, $\omega$ is the vorticity, and $x$ is the position vector within a plane. A schematic illustration of vorticity and helicity is shown in Figure 5.

Planimetry of the short-axis stack was performed with the Extended MR Workspace (version 2.6.3.5, Philips Healthcare, Best, Netherlands).to quantify myocardial mass. To compare HLHS patients' peak effective torsion and peak helicity density with the healthy controls', a Wilcoxon signed rank test was used. Correlations were studied using Spearman's rank correlation coefficient.

\section{Results}

MRI scans were performed in on HLHS subjects (29 male, 13 female) with a median age of 4.9 years (2.9-17.0 years) after establishing the Fontan circulation, and 11 healthy controls (4 male, 7 female) with a mean age of 12.1 (4.0-41.6). The subjects' demographic characteristics are listed in Table 1.

Effective torsion, helicity density of the (neo)aortic root of patients and controls and RV mass are summarized in Table 2. Peak effective torsion was significantly higher in HLHS patients compared to controls (Wilcoxon signed rank test, $\mathrm{P}=0.02$; Figure $6 A$ ). In all HLHS subjects, peak 


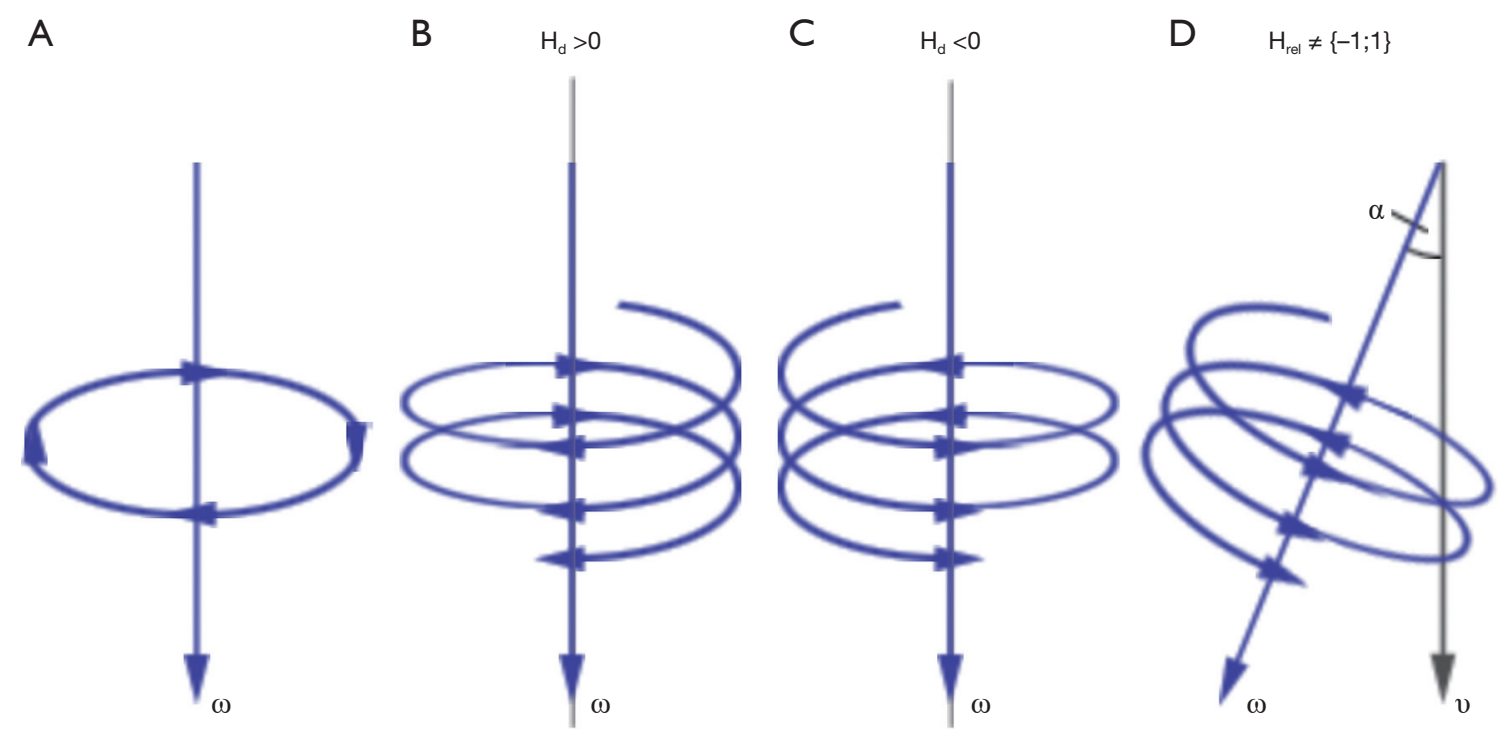

Figure 5 Illustration of vortex strength, helicity density and relative helicity. (A) Schematic illustration of vortex strength. (B) Helicity density is positive when it has a clockwise sense. (C) Helicity density is negative in case of the helix' counterclockwise sense. (D) Relative helicity. The vortex strength vector lies on the axis of the helix. $\omega$ : vortex strength; v: flow velocity.

Table 2 Geometric and fluid dynamics parameters in patients and healthy controls

\begin{tabular}{lccc}
\hline Parameter & HLHS subjects $(\mathrm{n}=42)$ & Healthy controls $(\mathrm{n}=11)$ & $\mathrm{P}$ value \\
\hline Peak effective torsion $\left(\mathrm{L} / \mathrm{cm}^{2}\right)$ & $0.112 \pm 0.053$ & $0.068 \pm 0.023$ & 0.04 \\
Peak helicity density $\left(\mathrm{m} / \mathrm{s}^{2}\right)$ & $24.41 \pm 31.91$ & $3.27 \pm 21.17$ & 0.03 \\
Myocardial mass & $67.88 \pm 25.75$ & - & - \\
\hline
\end{tabular}

HLHS, hypoplastic left heart syndrome.
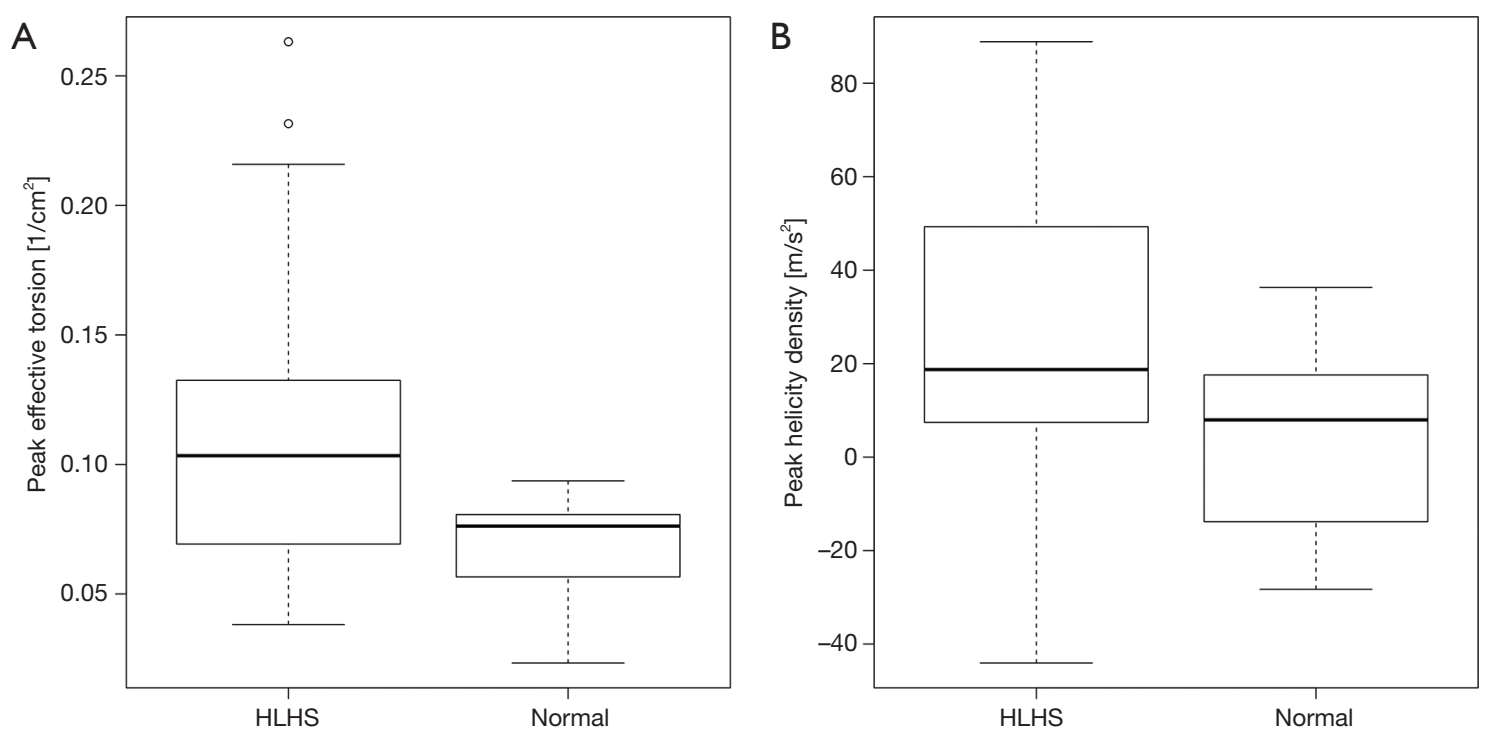

Figure 6 Comparison of HLHS patients with healthy controls. (A) Peak effective torsion is significantly higher in HLHS patients than in controls $(\mathrm{P}=0.02)$; (B) peak helicity density is significantly higher in HLHS patients than in controls $(\mathrm{P}=0.03)$. HLHS, hypoplastic left heart syndrome. 

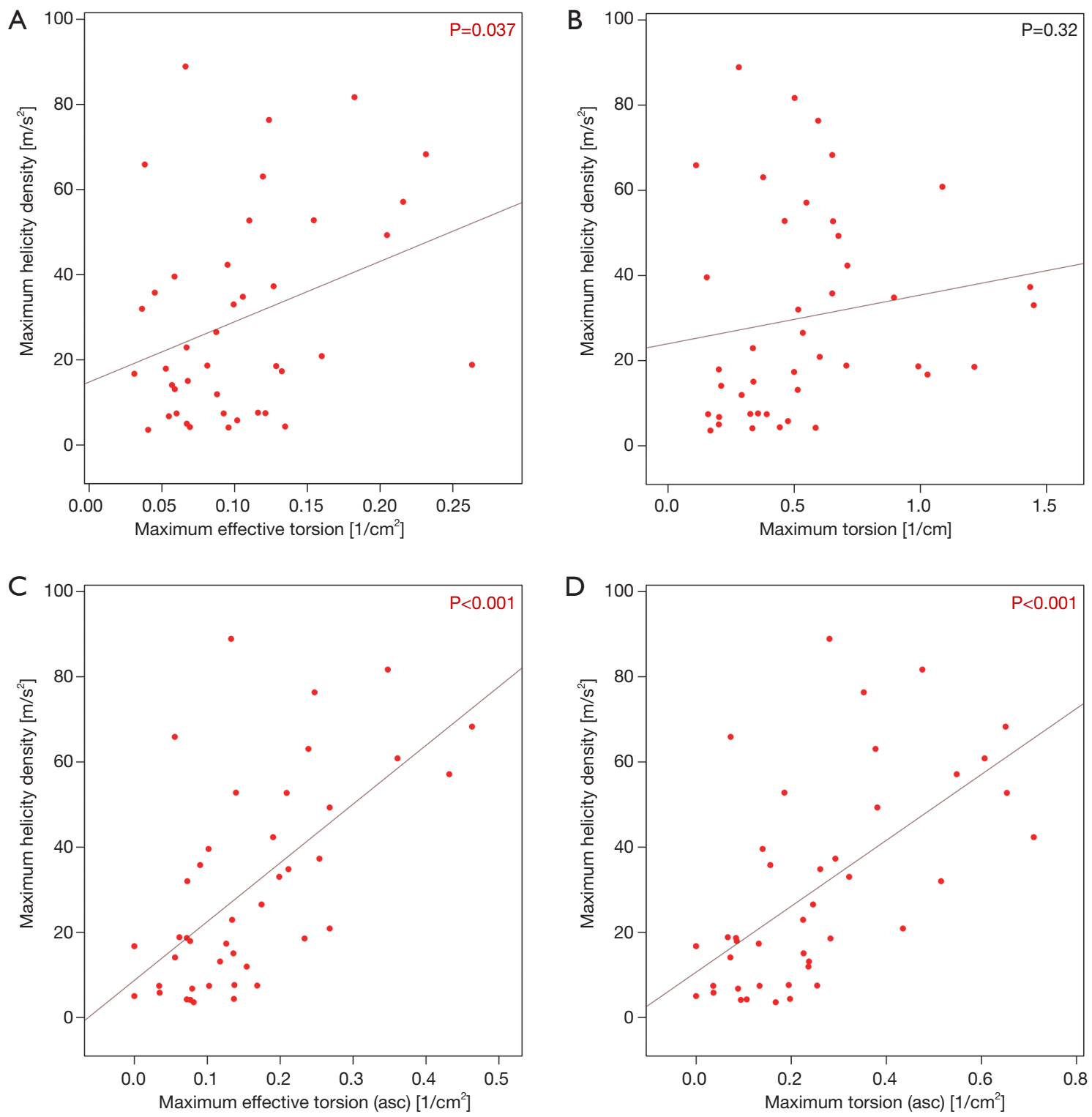

Figure 7 Relation of peak helicity density to torsion in the neoaorta of HLHS patients. (A,C) Use effective torsion, (A,B) show maximum torsion values along the aorta between valve and diapragm. Torsion values in (C,D) were calculated for the ascending section of the aorta. (A,C) Effective torsion, (B,D) conventional torsion.

effective torsion had a positive sign, corresponding to a path with clockwise rotation in forward direction. Compared to healthy controls, peak helicity density was significantly higher in HLHS patients (Wilcoxon signed rank test, $\mathrm{P}=0.03$; Figure 6 B). In $79 \%$ of HLHS patients versus $64 \%$ of controls, helical blood flow rotated clockwise according to positive peak helicity density values. Both, peak effective torsion and peak helicity density showed large variance for HLHS patients compared to controls reaching particularly large maximum values.

Maximum helicity density of blood flow in the entire aortic section correlated strongly with the maximum effective geometrical torsion of the ascending neoaorta (Spearman's $\rho=0.62, \mathrm{P}<0.001$ ) and moderately with the effective torsion of the entire aortic section (Spearman's $\rho$ $=0.35, \mathrm{P}=0.02)$ as shown in Figure 7 . Peak helicity density in the entire aortic section correlated moderately with the peak effective torsion of the ascending neoaorta (Spearman's $\rho$ 

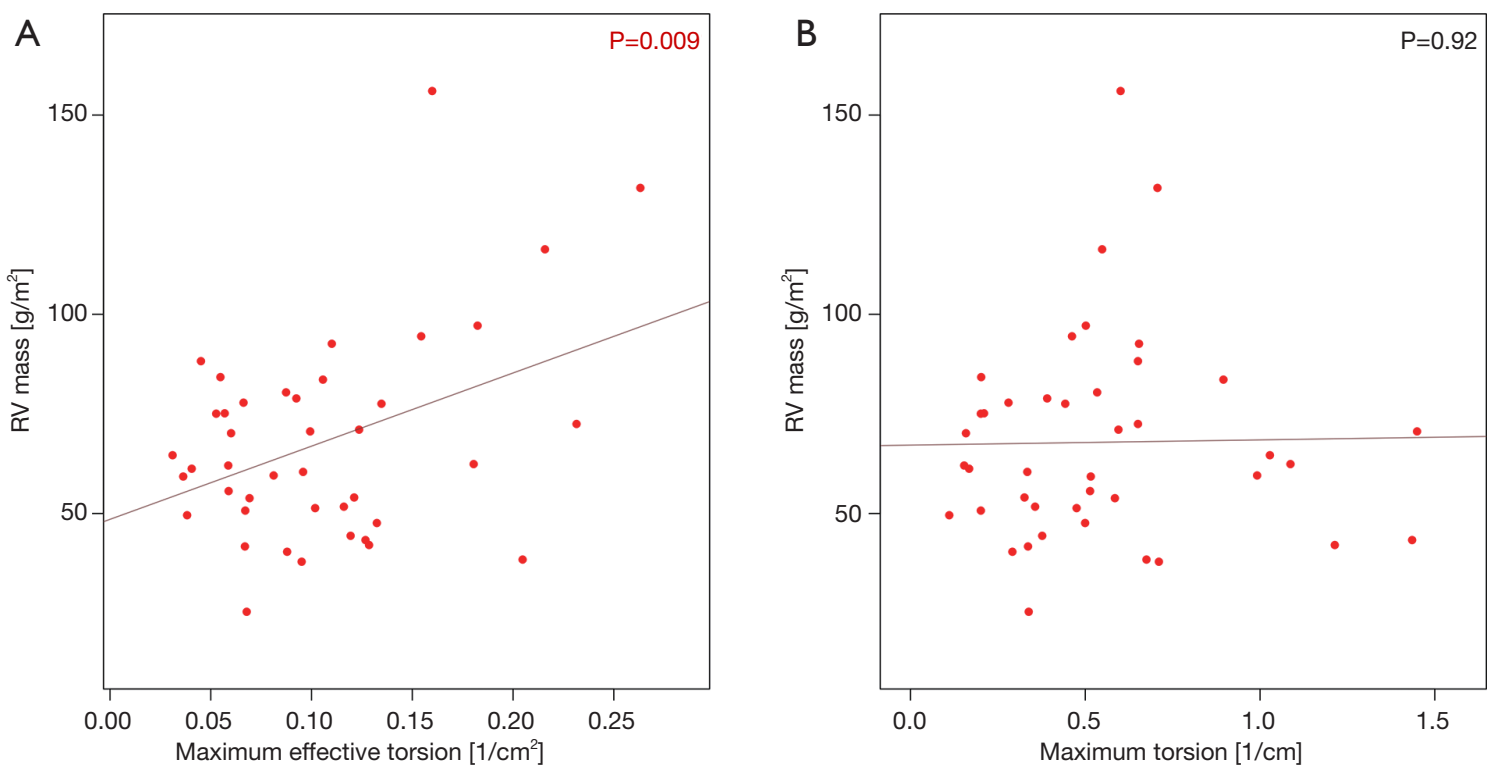

Figure 8 Relationship between RV myocardial mass with maximum effective torsion (A) and maximum torsion (B) in HLHS patients. The $\mathrm{P}$ values come from the Spearman test of correlations. RV, right ventricle; HLHS, hypoplastic left heart syndrome.

$=0.36, \mathrm{P}=0.02)$. The correlation of the peak helicity density in the entire aortic segment with the effective torsion peak of the entire aortic section was not statistically significant (Spearman's $\rho=0.19, \mathrm{P}=0.2$ ).

RV myocardial mass was $67.88 \pm 25.75 \mathrm{~g} / \mathrm{m}^{2}$ BSA and correlated with maximum effective torsion (Spearman's $\rho$ $=0.42, \mathrm{P}<0.01)$, as shown in Figure 8 .

\section{Discussion}

This study for the first time shows that in HLHS patients with Fontan circulation the abnormally high twist and effective torsion (Figure 2) of the neo-aorta after surgical reconstruction during Norwood operation (Figure 1) induces helical flow patterns (Figures 3,5). The results of our study suggest that patients with strong neoaortic torsion could be at an increased risk to develop hypertrophy.

Aortic twist can be quantified by geometric torsion, a quantity that may reach high values in sections of low curvatures. To mitigate that, we used the effective torsion as a novel index, which considers both torsion and vessel curvature, to characterizes the torsion of the aorta with a focus on regions with high curvature. A previous computational fluid dynamics study suggested that in aortic models, helical flow patterns with dominant preferential direction of rotation in the aorta are related to aortic twist (10). In fluid dynamics, secondary flow patterns with internal stresses, e.g., helical flow patterns, often indicate of energy loss due to resistance to the flow (13). Only few studies have previously investigated ventricular remodeling in HLHS patients and its association to secondary flow patterns induced by the abnormal neoaortic anatomy $(3,11,14)$.

According to previous studies $(10,15)$, curved vascular segments with no significant torsion generate symmetric, anti-directional helices, known as "Dean flow patterns", with increasing dominance of a particular direction of rotation when torsion increases. By averaging the crosssectional helicity density, symmetric anti-directional helices with different signs attenuate each other, which makes helicity density an appropriate index to determine the single helix formation or formations with dominant single helical flow patterns.

The significant correlation of neoaorta's effective torsion with helicity density (Figure 7) and myocardial mass (Figure 8) indicates a consecutive hypertrophy of the right ventricle (RV) to compensate for the increased flow resistance.

Future studies need to investigate whether modifications of aortic arch reconstruction techniques in HLHS, such the chimney reconstruction without patch (16) or arch angle augmentation (17), may change blood flow patterns. Furthermore, it has to be explored if other factors, such as the reduced elasticity of the neo-aorta $(5,18)$ is linked to the formation of abnormal flow pattern. This study may also 
stimulate others to conduct 4D-flow MRI or CFD studies to help to improve our understanding of aortic biophysical properties after extensive surgical reconstruction.

\section{Conclusions}

The abnormal twist of the HLHS patients' reconstructed neoaorta is associated with unfavorable changes in the aortic fluid dynamics and secondary ventricular hypertrophy. The findings of the present study may ultimately motivate modifications of Norwood procedure.

\section{Acknowledgments}

The authors thank Mrs. Traudel Hansen for her support with patient management. We would like to thank cand. med. Eva Rickers for graphic artwork.

Funding: This work was partially supported by an experienced researcher award from the Alexander von Humboldt Foundation to Prof. Kheradvar.

\section{Footnote}

Provenance and Peer Review: This article was commissioned by the Guest Editors (Yskert von Kodolitsch, Harald Kaemmerer, Koichiro Niwa) for the series "Current Management Aspects in Adult Congenital Heart Disease (ACHD): Part IV" published in Cardiovascular Diagnosis and Therapy. The article has undergone external peer review.

Reporting Checklist: The authors have completed the MDAR reporting checklist. Available at http://dx.doi.org/10.21037/ cdt-20-770

Data Sharing Statement: Available at http://dx.doi. org/10.21037/cdt-20-770

Conflicts of Interest: All authors have completed the ICMJE uniform disclosure form (available at http:// dx.doi.org/10.21037/cdt-20-770). The series "Current Management Aspects in Adult Congenital Heart Disease (ACHD): Part IV" was commissioned by the editorial office without any funding or sponsorship. Dr. Kheradvar reports grants from Humboldt Foundation, during the conduct of the study. The authors have no other conflicts of interest to declare.

Ethical Statement: The authors are accountable for all aspects of the work in ensuring that questions related to the accuracy or integrity of any part of the work are appropriately investigated and resolved. The study was conducted in accordance with the Declaration of Helsinki (as revised in 2013). The study was approved by the institutional ethics board of Kiel University's Faculty of Medicine (A168/07). The patient's ascent and the parent's consent were obtained prior to any MRI study.

Open Access Statement: This is an Open Access article distributed in accordance with the Creative Commons Attribution-NonCommercial-NoDerivs 4.0 International License (CC BY-NC-ND 4.0), which permits the noncommercial replication and distribution of the article with the strict proviso that no changes or edits are made and the original work is properly cited (including links to both the formal publication through the relevant DOI and the license). See: https://creativecommons.org/licenses/by-nc-nd/4.0/.

\section{References}

1. Norwood WI, Lang P, Hansen DD. Physiologic repair of aortic atresia-hypoplastic left heart syndrome. N Engl J Med 1983;308:23-6.

2. Chang AC, Farrell PE, Murdison KA, et al. Hypoplastic left heart syndrome: hemodynamic and angiographic assessment after initial reconstructive surgery and relevance to modified Fontan procedure. J Am Coll Cardiol 1991;17:1143-9.

3. Schäfer M, DiMaria MV, Jaggers J, et al. Suboptimal neoaortic arch geometry correlates with inefficient flow patterns in hypoplastic left heart syndrome. J Thorac Cardiovasc Surg 2019;158:e113-6.

4. Haller C, Chetan D, Saedi A, et al. Geometry and growth of the reconstructed aorta in patients with hypoplastic left heart syndrome and variants. J Thorac Cardiovasc Surg 2017;153:1479-87.e1.

5. Voges I, Jerosch-Herold M, Hedderich J, et al. Maladaptive aortic properties in children after palliation of hypoplastic left heart syndrome assessed by cardiovascular magnetic resonance imaging. Circulation 2010;122:1068-76.

6. Dyverfeldt P, Bissell M, Barker AJ, et al. 4D flow cardiovascular magnetic resonance consensus statement. J Cardiovasc Magn Reson 2015;17:72-90.

7. Bollache E, van Ooij P, Powell A, et al. Comparison of $4 \mathrm{D}$ flow and 2D velocity-encoded phase contrast MRI sequences for the evaluation of aortic hemodynamics. Int J Cardiovasc Imaging 2016;32:1529-41. 
8. Voges I, Scheewe J, Attmann T, et al. Abnormal aortic arch shape and vortical flow patterns are associated with descending aortic dilatation in patients with hypoplastic left heart syndrome. Int J Cardiol 2020:S01675273(20)33842-0.

9. Tse KM, Chang R, Lee HP, et al. A computational fluid dynamics study on geometrical influence of the aorta on haemodynamics. Eur J Cardiothorac Surg 2013;43:829-38.

10. Liu X, Fan Y, Deng X, et al. Effect of Spiral Flow on Transport of Oxygen in the Aorta: A Numerical Study. Ann Biomed Eng 2010;38:917-26.

11. Bruse JL, Cervi E, McLeod K, et al. Modeling of Congenital Hearts Alliance (MOCHA) Collaborative Group. Looks Do Matter! Aortic Arch Shape After Hypoplastic Left Heart Syndrome Palliation Correlates With Cavopulmonary Outcomes. Ann Thorac Surg 2017;103:645-54.

12. Bove EL, Mosca RS. Surgical repair of the hypoplastic left heart syndrome. Hypoplastic Left Heart Syndrome 1996;5:23-35.

13. Kheradvar A, Pedrizzetti G. Vortex Formation in the

Cite this article as: Gabbert DD, Trotz P, Kheradvar A, Jerosch-Herold M, Scheewe J, Kramer HH, Voges I, Rickers C. Abnormal torsion and helical flow patterns of the neo-aorta in hypoplastic left heart syndrome assessed with 4D-flow MRI. Cardiovasc Diagn Ther 2021;11(6):1379-1388. doi: 10.21037/ cdt-20-770
Cardiovascular System. Springer, 2012.

14. Biglino G, Giardini A, Ntsinjana HN, et al. Ventriculoarterial coupling in palliated hypoplastic left heart syndrome: noninvasive assessment of the effects of surgical arch reconstruction and shunt type. J Thorac Cardiovasc Surg 2014;148:1526-33.

15. Yamamoto K, Aribowo A, Hayamizu Y, et al. Visualization of the flow in a helical pipe. Fluid Dyn Res 2002;30(251).

16. Asada S, Yamagishi M, Itatani K, et al. Chimney reconstruction of the aortic arch in the Norwood procedure. J Thorac Cardiovasc Surg 2017;154:e51-4.

17. Hasegawa T, Yoshihiro O, Ayako M, et al. Aortic arch geometry after the Norwood procedure: The value of arch angle augmentation. J Thorac Cardiovasc Surg 2015;150:358-66.

18. Logoteta J, Ruppel C, Hansen JH, et al. Ventricular function and ventriculo-arterial coupling after palliation of hypoplastic left heart syndrome: A comparative study with Fontan patients with LV morphology. Int J Cardiol 2017;227:691-7. 